\title{
Subwavelength imaging with opaque left-handed nonlinear lens
}

\author{
Alexander A. Zharov ${ }^{1,2}$, Nina A. Zharova ${ }^{1,3}$, Ilya V. Shadrivov ${ }^{1}$ and Yuri S. Kivshar ${ }^{1}$ \\ ${ }^{1}$ Nonlinear Physics Group, Research School of Physical Sciences and Engineering, \\ Australian National University, Canberra ACT 0200, Australia \\ ${ }^{2}$ Institute for Physics of Microstructures, Russian Academy of Sciences, Nizhny Novgorod 603950, Russia \\ ${ }^{3}$ Institute of Applied Physics, Russian Academy of Sciences, Nizhny Novgorod 603600, Russia
}

\begin{abstract}
We introduce the concept of subwavelength imaging with an opaque nonlinear left-handed lens by generating the second-harmonic field. We consider a slab of composite left-handed metamaterial with quadratic nonlinear response and show that such a flat lens can form, under certain conditions, an image of the second-harmonic field of the source being opaque at the fundamental frequency.
\end{abstract}

PACS numbers: 41.20.Jb, 42.25.Bs, 78.20.Ci, 42.70.Qs

One of the most unique properties of the recently demonstrated left-handed metamaterials, i.e. materials with simultaneously negative real parts of dielectric permittivity and magnetic permeability [1], is their ability to focus electromagnetic waves by a flat slab of the material, the property which makes these materials quite different from the conventional optical lenses with the positive refractive index needed to have curved surfaces to form an image. Recently, Pendry [2] argued that a slab of a lossless left-handed material with $\epsilon=\mu=-1$ should behave like a perfect lens enabling to obtain an ideal image of a point source through the amplification of the evanescent components of the field.

While recent experimental demonstrations confirmed the main features of negative refraction of the left-handed materials [3, 4], the question of near-perfect imaging by flat lens and near-field focusing still remain highly controversial [5], and it severely constrained because of large dissipation and dispersion of metamaterials. Nevertheless, numerical studies indicate [6] that nearly-perfect imaging should be expected even under realistic conditions when both dispersion and losses of the left-handed composites are taken into account. More importantly, some of the properties of the left-handed materials, such as negative refraction, have been predicted [7] and recently demonstrated experimentally $[8]$ in photonic crystals, which are inhomogeneous periodic structures with a lattice constant comparable to the wavelength. This negative refraction allows considerable control over light propagation, and it opens up doors for new applications of microstructured materials and the extension of the basic concepts from microwaves to optical frequencies.

Until now, all properties of flat lenses and subwavelength imaging have been studied only for linear waves. However, it has been already noticed that the left-handed metamaterials may possess quite complicated nonlinear magnetic response [9], or properties of such materials can be altered by inserting diodes in the resonators of the composite structure thus making the response of the entire material nonlinear [10. In this Letter, we make one step forward into this direction and study the effects of the second-harmonic generation and subwavelength imaging by a nonlinear left-handed lens. In particular, we analyze the imaging properties of a slab of metamaterial with quadratic nonlinear response and demonstrate, both analytically and numerically, that such a slab can form an image of the second-harmonic field of the source being opaque at the fundamental frequency. This can happen under certain conditions, which include Pendry's conditions of a perfect lens satisfied for the second-harmonic field, $\epsilon(2 \omega)=\mu(2 \omega)=-1$, leading to the conditions for $\epsilon(\omega)$ and $\mu(\omega)$ being of the opposite signs at the fundamental frequency $\omega$. More importantly, for the case of two sources we show that the resolution of such a nonlinear left-handed flat lens can be made indeed better than the radiation wavelength.

We consider a lens in the form of a layer of left-handed metamaterial with the thickness $D$, as shown schematically in Fig. 1. We assume that the metamaterial is a three-dimensional composite structure made of wires and split-ring resonators (SRRs) in the form of a cubic lattice. When the lattice period $d$ is much smaller than the radiation wavelength $\lambda$, i.e. $d \ll \lambda$, this composite structure can be described within the effective-medium approximation, and it can be characterized by dielectric permittivity and magnetic permeability which, for the specific structure and in the linear regime can be derived consistently and can be written in the form

$$
\begin{gathered}
\epsilon(\omega)=1-\frac{\omega_{p}^{2}}{\omega\left(\omega-i \gamma_{e}\right)}, \\
\mu(\omega)=1+\frac{F \omega^{2}}{\omega_{0}^{2}-\omega^{2}+i \gamma_{m} \omega},
\end{gathered}
$$

where $\omega_{p}=\sqrt{2 \pi c^{2} / d^{2} \ln (d / r)}$ is the effective plasma frequency, $\omega_{0}=\bar{\omega}_{0} \sqrt{(1-F)}, \bar{\omega}_{0}$ is the eigenfrequency of an isolated SRR, $F$ is the volume density of SRRs, $\gamma_{e}$ and $\gamma_{m}$ are the damping coefficients, $\omega$ is the frequency of the external electromagnetic field, $r$ is the wire radius, and $c$ is the speed of light. In the frequency range where the real parts of $\epsilon$ and $\mu$ are both negative and for $\gamma_{e}, \gamma_{m} \ll \omega$, such a composite structure demonstrates left-handed transmission, whereas for $\omega<\omega_{0}$, it is opaque because the signs of $\epsilon$ and $\mu$ are opposite. 


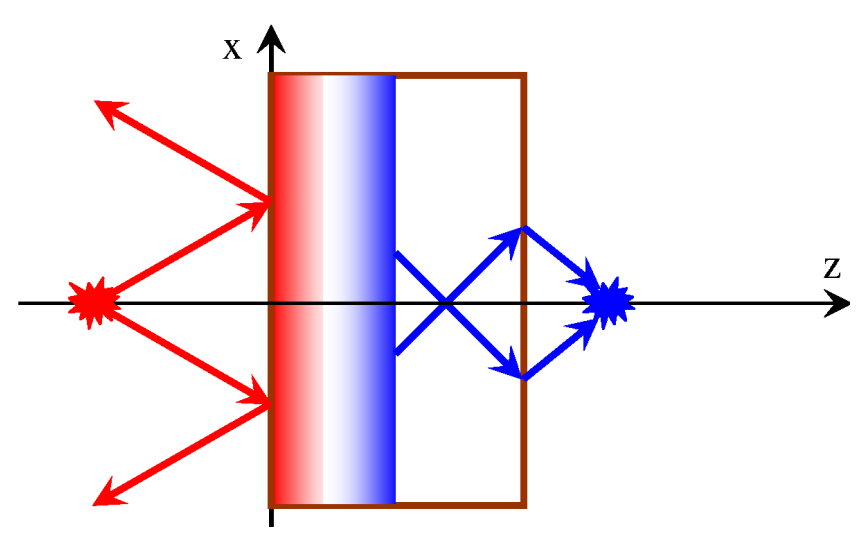

FIG. 1: Schematic of the problem. Electromagnetic waves emitted by a source (located at $z=-z_{s}$ ) are reflected from an opaque slab of a left-handed material. Inside the slab, the exponentially decaying field at the fundamental frequency $\omega$ generates the second-harmonic field at $2 \omega$, which penetrates through the slab creating an image at the second harmonics.

In order to achieve a nonlinear response in such a structure, we assume that each SRR includes a nonlinear element, e.g. a diode inserted in the SRR [10]. If the diode has an asymmetric current-voltage characteristics, the unit cell does not possess a center of symmetry with respect to the field direction, and the resulting nonlinear response should include, in particular, the second harmonic of the source frequency.

Our idea is to satisfy the perfect-lens conditions for the second-harmonic waves and, therefore, we take $\mu(2 \omega)=$ $\epsilon(2 \omega)=-1$. From Eqs. (1), (2) we can find that these conditions can be satisfied provided

$$
\epsilon(\omega)=-7, \quad \mu(\omega)=(3-F) /(3-2 F) .
$$

For this choice of the material parameters, the slab is opaque at the fundamental frequency $\omega$, and the waves do not penetrate into it. However, the nonlinear quadratic response of the material can allow the process of the second-harmonic generation. Since the material is transparent at the frequency $2 \omega$, we expect that the secondharmonic field can propagate through the slab creating an image of the source behind the flat lens.

Using the so-called undepleted pump approximation, we can obtain the equation for the TM-polarized secondharmonic field $H_{y}^{(2 \omega)}(x, z)$ inside the slab, which has the form well-known in the theory of the second-harmonic generation (see e.g. Ref. [1]])

$$
\Delta H_{y}^{(2 \omega)}+K^{2}(2 \omega) H_{y}^{(2 \omega)}=-\frac{16 \pi \omega^{2} \epsilon(2 \omega)}{c^{2}} M_{N L}^{(2 \omega)},
$$

where $\Delta$ is the Laplacian acting in the space $(x, z)$, $K^{2}(2 \omega)=4 k_{0}^{2} \epsilon(2 \omega) \mu(2 \omega)$, and $M_{N L}^{(2 \omega)}$ is the nonlinear magnetization of the unit volume of the metamaterial at the frequency $2 \omega$, which appears due to the nonlinear magnetic momentum of SRR,

$$
M_{N L}^{(2 \omega)}=-\frac{3}{4} \chi(\omega)\left[H_{y}^{(\omega)}(x, z)\right]^{2}
$$

where

$$
\chi(\omega)=\frac{\left(\pi a^{2}\right)^{3} \omega_{0}^{4}}{c^{3} d^{3} U_{c} R_{d} \omega^{2}}\left[\left(\frac{\omega_{0}^{2}}{\omega^{2}}-1\right)^{2}-i \frac{\gamma_{m}}{\omega}\left(\frac{\omega_{0}^{2}}{\omega^{2}}+2\right)\right]^{-1},
$$

$H_{y}^{(\omega)}(x, z)$ is the spatial distribution of the magnetic field at the fundamental frequency in the slab, $a$ is the radius of the resonator rings, $R_{d}$ is the differential resistance of the diode at zero voltage, $k_{0}=\omega / c$, and $U_{c}$ is the diode parameter defined from the current-voltage characteristics of the diode which we take in the form $I=I_{0}\left(e^{U / U_{c}}-1\right)$. The right-hand side of Eq. (4) vanishes outside the metamaterial slab.

Applying the Fourier transform along the $x$ direction, we obtain the equation for the function $\bar{H}_{y}^{(2 \omega)}=$ $\bar{H}_{y}^{(2 \omega)}\left(k_{x}, z\right)$ in terms of $\bar{H}_{y}^{(\omega)}=\bar{H}_{y}^{(\omega)}\left(k_{x}, z\right)$ and $G=$ $G\left(k_{x}, z\right)$ which are the Fourier transforms of $H^{(\omega)}(x, z)$, $H^{(2 \omega)}(x, z)$ and $\left[H^{(\omega)}(x, z)\right]^{2}$, respectively:

$$
\frac{d^{2} \bar{H}_{y}^{(2 \omega)}}{d z^{2}}+\left[K^{2}(2 \omega)-4 k_{x}^{2}\right] \bar{H}_{y}^{(2 \omega)}=\eta G,
$$

where $\eta=12 \pi k_{0}^{2} \epsilon(2 \omega) \chi(\omega)$. Using the convolution theorem, we express the function $G$ through the spectrum of the magnetic field at the fundamental frequency $\bar{H}_{y}^{(\omega)}$ in the form

$$
G=\int_{-\infty}^{\infty} \bar{H}^{(\omega)}\left(k_{x}^{\prime}, z\right) \bar{H}^{(\omega)}\left(k_{x}-k_{x}^{\prime}, z\right) d k_{x}^{\prime} .
$$

Within the framework of the undepleted pump approximation, the function $\bar{H}^{(\omega)}\left(k_{x}, z\right)$ can be found as a solution of the linear problem describing the electromagnetic field at the fundamental frequency transmitted into the left-handed slab,

$$
\bar{H}^{(\omega)}=\frac{2 \kappa_{1} e^{-i k_{0} \kappa_{1} z_{s}}}{D_{s}\left(\omega, k_{x}\right)}\left[Z_{1} e^{k_{0} \kappa_{2} z}-Z_{2} e^{k_{0} \kappa_{2}(2 D-z)}\right] S(\gamma),
$$

where $S(\gamma)$ is the spectral function of the source at the fundamental frequency located at the distance $z_{s}$ from the left-handed slab, $\kappa_{1}=\sqrt{1-\gamma^{2}}, \kappa_{2}=$ $\sqrt{\gamma^{2}-\epsilon(\omega) \mu(\omega)}, \gamma=k_{x} / k_{0}, Z_{1,2}=\kappa_{1} \pm i \kappa_{2} / \epsilon(\omega)$, and $D\left(\omega, k_{x}\right)=Z_{1}^{2}-Z_{2}^{2} \exp \left(2 k_{0} \kappa_{2} D\right)$. The spectral function $S(\gamma)$ includes both fast propagating $(\gamma \leq 1)$ and slow evanescent $(\gamma>1)$ spatial harmonics. Possible distortions of the second-harmonic image can be caused by the pre-exponential factor in Eq. (8), and the main effect is due to the pole singularity defined by the equation

$$
D\left(\omega, k_{x}\right)=0
$$

that characterizes the resonant excitation of surface polaritons which are known to limit the resolution of a lefthanded lens. In the case of a thick slab, i.e. $k_{0} \kappa_{2} D \gg 1$, the resonant wavenumber of the surface waves can be found in the form

$$
\gamma_{\mathrm{sp}}^{2}=\frac{\epsilon(\omega)[\epsilon(\omega)-\mu(\omega)]}{\epsilon^{2}(\omega)-1} .
$$


Substituting the explicit expressions for $\epsilon(\omega)$ and $\mu(\omega)$ from Eq. (3) into Eq. (10), we obtain a simple estimate for an expected resolution limit of the nonlinear left-handed lens in terms of the critical (limiting) wavenumber,

$$
\gamma_{\lim }^{2} \approx 1+\frac{(3-F)}{7(3-2 F)} .
$$

However, the existence of this critical wavenumber does not necessarily limits the lens resolution and, in reality, the effect of surface waves on the imaging properties of the nonlinear lens depends on the efficiency of their excitation by each particular source.

Analytical solution of the problem for the spatial spectrum of the second-harmonic field transmitted through the left-handed slab can be obtained for narrow enough spectrum of the source, i.e., when the width of the source spectrum at the fundamental frequency does not exceed the value $\gamma_{c}$, where

$$
\gamma_{c}^{2} \approx|\epsilon(\omega) \mu(\omega)| .
$$

Then, we can use the impedance boundary conditions for the fundamental field at the interface between vacuum and the metamaterial slab at $z=0$. Subsequent numerical results indicate that this approximation remains valid provided $\gamma_{c} \gg \gamma_{\text {lim }}$.

To solve the problem analytically, we assume that the wave at the fundamental frequency $\omega$ penetrates inside the slab on a distance (the skin layer) much smaller than the slab thickness $D$, i.e. $D \delta \gg 1$, where $\delta=$ $k_{0} \sqrt{-\epsilon(\omega) \mu(\omega)}$. Taking into account the actual values of $\epsilon(\omega)$ and $\mu(\omega)$ (3), one can see that the fundamental frequency penetration depth, $\sim(2 \pi \delta)^{-1}$, does not exceed $\lambda / 17$. Then, Eq. (6) can be re-written in the form

$$
\frac{d^{2} \bar{H}_{y}^{(2 \omega)}}{d z^{2}}+\left[K^{2}(2 \omega)-4 k_{0}^{2} \gamma^{2}\right] \bar{H}_{y}^{(2 \omega)}=\eta e^{-2 \delta z} A_{0}(\gamma),
$$

where $A_{0}(\gamma)=\int_{-\infty}^{\infty} \xi\left(\gamma^{\prime}\right) \xi\left(\gamma-\gamma^{\prime}\right) d \gamma^{\prime}$, and $\xi(\gamma)=$ $e^{\delta z} \bar{H}^{(\omega)}(\gamma, z)$ does not depend on $z$. As a result, the general solution for the second harmonic inside the slab can be presented in the form

$$
\bar{H}_{y}^{(2 \omega)}(\gamma, z)=C_{1} e^{2 k_{0} \kappa_{2} z}+C_{2} e^{-2 k_{0} \kappa_{2} z}+C_{3} e^{-2 \delta z},
$$

where $C_{1,2}$ are two constants which should be determined from the boundary conditions, and

$$
C_{3}=\left(\frac{1}{4 k_{0}^{2}}\right) \frac{\eta A_{0}(\gamma)}{[\epsilon(2 \omega) \mu(2 \omega)-\epsilon(\omega) \mu(\omega)]} .
$$

We should satisfy the continuity of the tangential components of the magnetic $H_{y}^{(2 \omega)}$ and electric $E_{x}^{(2 \omega)}$ fields at the interfaces between air and the metamaterial slab, i.e. at $z=0$ and $z=D$. As a result, we obtain the second harmonic of the magnetic field behind the slab (for $z>D$ ) where the image is expected to form,

$$
\bar{H}_{y}^{(2 \omega)}(\gamma, z)=-\frac{1}{2}\left\{1-i \frac{\kappa_{2} \epsilon(\omega)}{\kappa_{1} \epsilon(2 \omega)}\right\} C_{3} e^{2 k_{0} \kappa_{1}(2 D-z)} .
$$

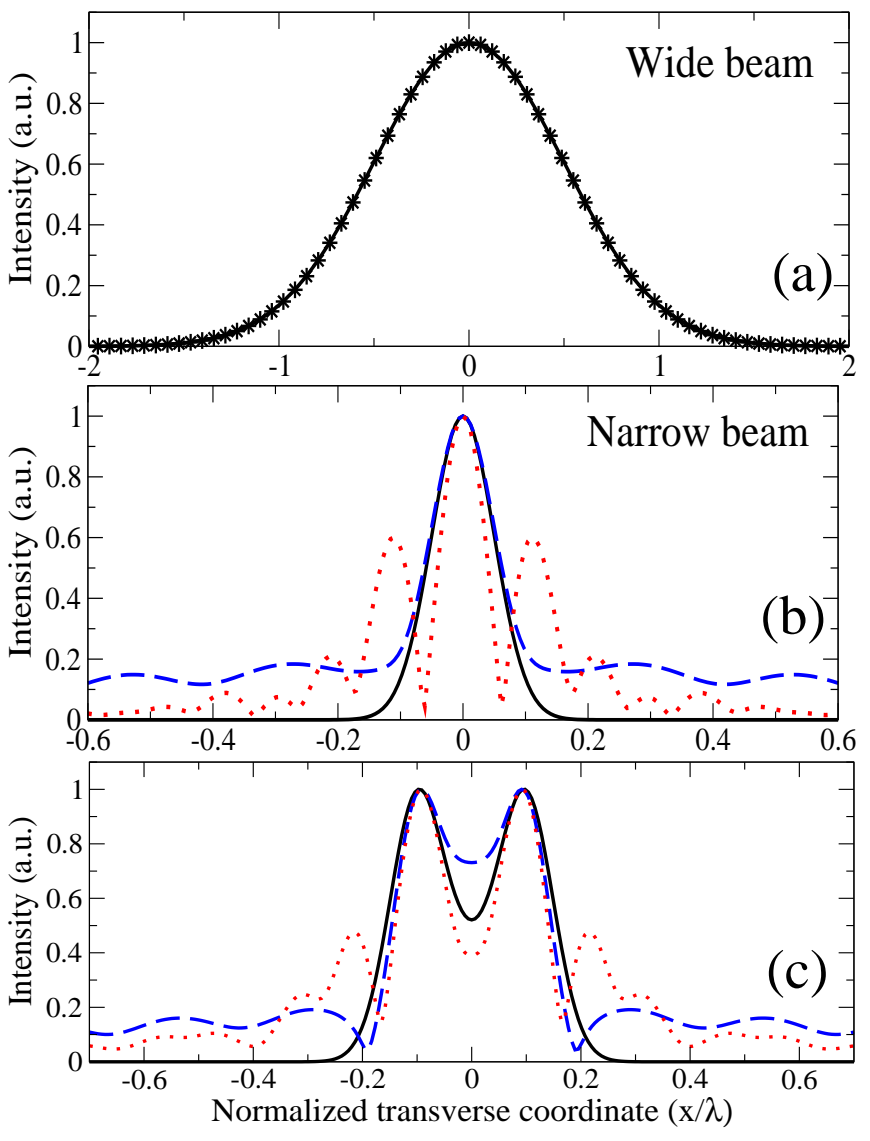

FIG. 2: Numerical results for imaging by a nonlinear lefthanded lens. Shown are the intensities of the fundamental field at the source location (solid) and the second-harmonic field at the image plane for different cases (stars, dashed and dotted lines). (a) Wide beam (the width is $\lambda$ ) generated by a single source, $D=\lambda, z_{s}=\lambda / 2$. (b) Narrow beam (the width is $\lambda / 10)$ generated by a single source; dashed-the image for $D=\lambda / 10, z_{s}=\lambda / 20$; dotted - the image for $D=0.3 \lambda$, $z_{s}=0.15 \lambda$. (c) Imaging by two sources separated by the distance $\lambda / 5$; dashed - the image for $D=\lambda / 10$ and $z_{s}=$ $0.03 \lambda$; dotted - the image for $D=0.3 \lambda$ and $z_{s}=\lambda / 5$.

For wide beams with narrow spectra, Eq. (15) can be rewritten in the form

$$
\begin{gathered}
\bar{H}_{y}^{(2 \omega)}(\gamma, z)=-\frac{\eta}{2 k_{0}^{2}[\epsilon(2 \omega) \mu(2 \omega)-\epsilon(\omega) \mu(\omega)]} \times \\
{\left[1-\frac{\sqrt{-\epsilon(\omega) \mu(\omega)}}{\epsilon(2 \omega)}\right]\left[1-i \frac{\sqrt{-\epsilon(\omega) \mu(\omega)}}{\epsilon(\omega)}\right]^{-1} \times} \\
\exp \left\{2 k_{0} \kappa_{1}\left(2 D-z-z_{s}\right)\right\} \int_{-\infty}^{\infty} S\left(\gamma^{\prime}\right) S\left(\gamma-\gamma^{\prime}\right) d \gamma^{\prime} .
\end{gathered}
$$

Thus, the squared field at the fundamental frequency acts as an effective source of the second-harmonic field and, as a result, the image of the squared field is reproduced by the nonlinear left-handed lens. This image appears at the point $z_{\mathrm{im}}=D-z_{s}$, and this result coincides with the corresponding result for the linear lens discussed earlier [2]. 


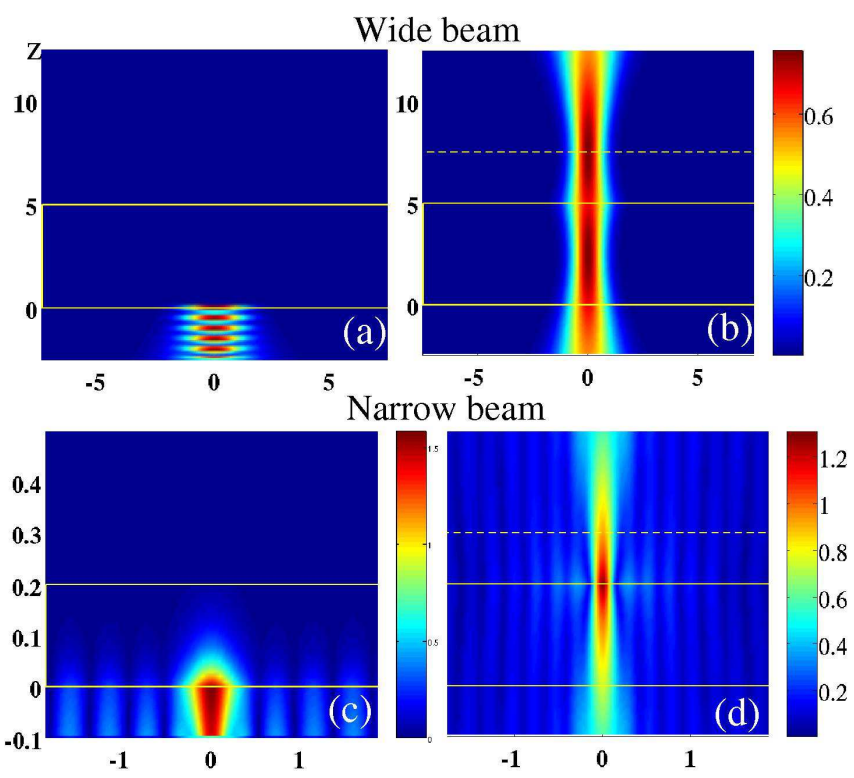

FIG. 3: Intensity of the fundamental $[(\mathrm{a}, \mathrm{c})]$ and secondharmonic $[(b, d)]$ beams (in units of the wavelength) for the problem of the second-harmonic generation and imaging by a nonlinear left-handed lens. (a,b) Wide beam $(D=5 \lambda$, $z_{s}=2.5 \lambda$, and $\left.a_{0}=\lambda\right)$ and $(\mathrm{c}, \mathrm{d})$ narrow beam $(D=\lambda / 5$, $z_{s}=\lambda / 10$, and $\left.a_{0}=\lambda / 4\right)$. Solid lines mark the flat surfaces of the nonlinear left-handed lens. Dashed lines show the predicted locations of the second-harmonic image.

When the size of the source is comparable or less than the wavelength $\lambda$ of the fundamental-frequency wave, the problem cannot be treated analytically, and Eq. (4) has been solved numerically. In Figs. 2(a-c), we present our numerical results for the intensity distribution of the incident beam at the source point and the field distribution of the second-harmonic beam at the image location, normalized to the field maxima. The actual amplitude of the electromagnetic field at the image location is lower than the amplitude of the source because of a finite efficiency of the process of the second-harmonic generation.
For the objects with the spatial scale larger or equal to the radiation wavelength, the second-harmonic field profile coincides with the intensity of the fundamental field generated by the source, as shown in Fig. 2(a).

However, when the source contains the spatial scales less than the radiation wavelength, the imaging properties of the nonlinear lens depend strongly on the slab thickness $D$. As an example, in Fig. 2(b) we show the results for the transmission of an incident Gaussian beam of the width $\lambda / 10$ which reproduces almost exactly the source profile at the image plane in the case of a thin lens (dashed line) but generates a strongly distorted image when the slab thickness becomes larger than a half of the wavelength $\lambda$. Distortions appear as periodic variation of the second-harmonic field being caused by excitation of surface waves. Intensity distribution of the magnetic field in the fundamental and second-harmonic fields are shown in Figs. 3(a-d) for $(a, b)$ large and $(c, d)$ small (compared to the radiation wavelength) size of the source, respectively.

Figure 2(c) shows the numerical results for imaging of two sources that generate the Gaussian beams with the maxima separated by the distance $\lambda / 5$ in the transverse direction. Again, the image reproduces very well the source for a thinner lens, and therefore a thin nonlinear lens does provide a subwavelength resolution of the second-harmonic field. In contrast to the linear flat lens, the resolution of the nonlinear lens depends on the distance $z_{s}$ between the source and the lens, and the optimal distance can be determined separately for each particular case.

In conclusion, we have studied the second-harmonic generation and subwavelength imaging by a nonlinear left-handed lens, i.e. a slab of metamaterial with quadratic nonlinear response. We have demonstrated, both analytically and numerically, that such a slab can form an image of the second-harmonic field of the source being opaque at the fundamental frequency, with the resolution that can be made indeed better than the radiation wavelength.
[1] V.G. Veselago, Usp. Fiz. Nauk 92, 517 (1967) [Sov. Phys. Usp. 10, 509 (1968)].

[2] J.B. Pendry, Phys. Rev. Lett. 85, 3966 (2000).

[3] R.A. Shelby, D.R. Smith, and S. Schultz, Science 292, 77 (2001).

[4] C.G. Parazzoli, R.B. Greegor, K. Li, B.E.C. Koltenbah, and M. Tanielian, Phys. Rev. Lett. 90, 107401 (2003).

[5] L. Venema, Nature (London) 420, 119 (2002).

[6] See, e.g., S.A. Cummer, Appl. Phys. Lett. 82, 1503 (2003); M. Feise and Yu.S. Kivshar, arXiv/physics: 0403111 (2004), and references therein.

[7] M. Notomi, Phys. Rev. B 62, 10696 (2002); Opt. Quantum Electron. 34, 133 (2002); A.L. Efros and A.L. Pokrovsky, Solid State Commun. 129, 643 (2004).
[8] E. Cubukcu, K. Aydin, E. Ozbay, S. Foteinopoulou, C.M. Soukoulis, Nature 423, 605 (2003); P.V. Parimi, W.T. Lu, P. Vodo, S. Sridhar, Nature 426, 404 (2003); P.V. Parimi, W.T. Lu, P. Vodo, J. Sokoloff, J.S. Derov, and S. Sridhar, Phys. Rev. Lett. 92, 127401 (2004).

[9] A.A. Zharov, I.V. Shadrivov, and Yu.S. Kivshar, Phys. Rev. Lett. 91, 037401 (2003).

[10] M. Lapine, M. Gorkunov, and K.H. Ringhofer, Phys. Rev. E 67, 065601 (2003).

[11] M. B. Vinogradova, O. V. Rudenko, and A. P. Sukhorukov, Theory of Waves (Nauka, Moscow, 1990) (in Russian). 